\title{
Las ciencias sociales y de la conducta: su problematización desde la complejidad
}

\section{Social and behavioral sciences: its problematization since the complexity}

Manuel Villarruel-Fuentes

Tecnológico Nacional de México - Instituto Tecnológico de Úrsulo Galván, Veracruz, México.

\section{Resumen}

Objetivo: analizar las teorías que explican los enfoques de la complejidad, así como el pensamiento que lo hace posible. Método: se recuperaron los aportes de Niklas Luhmann (1998), Rolando García (2011) y Edgar Morin (1994), estableciendo con ellos un comparativo que clarifica sus disensos y distinguiéndose posibles pautas de conciliación entre sus postulados al abordar las problemáticas sociales y humanas desde las premisas de un pensamiento complejo, cuyo método no es ajeno a las teorías asociadas al enfoque sistémico. Conclusión: Para alcanzar el nivel de discernimiento necesario en las ciencias sociales, humanas y de la conducta, es preciso entender que las lógicas tradicionales surgen como producto de acontecimientos históricos, de procesos sociales evolutivos condicionados por factores políticos y económicos que impactan fuertemente en la cultura.

Palabras clave: Complejidad, resiliencia, interdisciplina, transdisciplina, educación.

\section{Abstract}

Objective: to analyze the theories that explain the complexity approaches, as well as the thought that makes it possible. Method: the contributions of Niklas Luhmann (1998), Rolando García (2011) and Edgar Morin (1994) were recovered, establishing with them a comparative that clarifies their dissents, distinguishing possible patterns of conciliation among their postulates, addressing social and human problems from the premises of a complex thought, whose method is not alien to the theories associated with the systemic approach. Conclusion: In order to reach the necessary level of discernment within the social, human and behavioral sciences, it is necessary to understand that traditional logics arise as a product of historical events, of evolutionary social processes, conditioned by political and economic factors that strongly impact on culture.

Keywords: Complexity, resilience, interdiscipline, transdiscipline, education. 


\section{INTRODUCCIÓN}

Mucho se ha discutido la relevancia que la educación científica tiene para el desarrollo social y el crecimiento humano. Desde distintos ángulos y dimensiones su abordaje ha buscado conciliar los aspectos conceptuales y metodológicos que identifican a los paradigmas dominantes, ninguno tan ortodoxo como el experimental.

Sobre esta base se entiende el cúmulo de reflexiones epistemológicas que han acompañado al proceso de investigación científica a lo largo de la historia, cuyos disensos, además de teóricos, han sido ideológicos, al asociar sus sustentos argumentativos con los procesos civilizatorios dominantes.

Si bien algunos paradigmas han surgido como alternativas a las diversas crisis que la humanidad ha confrontado a lo largo del tiempo -propuestos como arquetipos o modelos emergentes-, su denominador común sigue ligado al descubrimiento o bien a la construcción de una realidad que se anhela conocer.

Sin embargo, desde finales del siglo XX los fundamentos teóricos que explican la realidad se han visto trastocados por la idea de un contexto problemático que anida en la complejidad y el caos, alimentados por un enfoque sistémico que asume que lo importante estriba en las relaciones que establecen las partes con respecto al todo.

Esto puede parecer una nimiedad en virtud de la cantidad de obras que sobre el tema se han escrito, aunque en esencia su aseveración implica una reflexión epistémica que confronta los principios que desde el siglo XVII han alimentado el pensamiento científico, centrados en la parcialidad o segmentación de los fenómenos objeto de estudio, que al ser estudiados analíticamente proyectan evidencias fraccionadas, cuya acumulación es capaz de representar una realidad isomórfica.

Al contrario de estos principios, perpetuados por una realidad que se desea explicar, el pensar los contextos problemáticos en términos sistémicos lleva ya implícito un abordaje definido en la complejidad, entendida no por los componentes que la integran, sino por las relaciones que estos pueden llegar a mostrar. Bajo esta nueva lógica de entendimiento se habla entonces de sistemas complejos, interpretada como una redundancia conceptual, en virtud de que todo sistema es complejo por antonomasia. Esto supera el sentido aditivo que durante siglos mantuvo la unidad simbólica del modelo científico tradicional, concebido desde una perspectiva convergente, asumiéndose el todo como una unión de elementos o partes ensamblables mediante el intelecto y la razón.

Ante ello es fácil advertir la inoperancia de estos principios cartesianos, cuya empiria pareció justificar la veracidad de aquello que se asumía como real. En esta vertiente las diferencias más conspicuas alcanzan el plano de lo metodológico, donde el paradigma 
empírico-analítico asumió un sentido técnico, netamente procedimental, mientras que desde la complejidad se entiende ahora en un plano epistemológico. Se trata de la vieja disputa entre la teoría y la práctica, recuperada ahora con fines de contraste.

Lo que aquí se señala no es la anulación del binomio teoría-práctica, sino su reconceptualización, a partir de la superación del reduccionismo disciplinar que distinguió a la ciencia de los siglos XIX y XX y que desembocó en una codependencia exigida con la tecnología, al grado de trasladar el significado original de esta relación a los nuevos espacios de entendimiento; hoy se habla de ciencia y tecnología como un ente indisoluble, usualmente incuestionable. Esta no es una dirección efectiva para los nuevos razonamientos emanados de la complejidad.

Pese a encontrar claridad en torno a lo que significa la complejidad, aún persisten serias dificultades para concretarla como vía de acceso a la realidad, sobre todo por los disensos, vacíos y contradicciones emanados de su operatividad. Al respecto existen quienes intentan aplicarla desde el tradicional baluarte de las disciplinas -ciencias-, o bien, desde la interdisciplina y la transdisciplina. En todos los casos persiste un fuerte arraigo con las acostumbradas estructuras mentales provenientes del pensamiento newtoniano.

Las circunstancias referidas dejan entrever una doble complejidad dentro de la ciencia: en primera instancia su entendimiento por quienes ya realizan investigación científica; por otra parte, su enseñanza dentro de los entornos escolares -espacio donde se forman los nuevos investigadores científicos-. Al respecto, el presente artículo se sitúa en medio del reto que significa plantear y delimitar fenómenos objeto de estudio, compendiados en contextos problemáticos que deben ser observados como sistemas, en busca de lograr una alfabetización diferente, novedosa, incluso emergente.

Se trata de una ciencia nueva, que encuentra diferentes reflexiones desde los conceptos de Von Bertalanffy, Heinz Pagels, Roger Lewin, Edgar Morin, Rolando García, Gaston Bachelard, François Jacob, Michel Serres, Michell Waldrop, Iliya Prigogine, Niklas Luhmann, Humberto Maturana, y desde luego Edward Lorenz, climatólogo norteamericano, considerado el impulsor de esta forma de pensamiento basado en el caos. Nuevo razonamiento que González (2009) sintetiza al señalar que:

Hay ecuaciones o sistemas de ecuaciones que a partir de ciertos valores de las variables, los valores que siguen resultan impredecibles, aperiódicos, se dice entonces que se ha llegado al caos determinista, determinista porque se somete, aún con las características citadas, a regularidades que se estudian y se tratan con métodos de las ciencias exactas, naturales y humanísticas. (243).

Se muestra así el denominado efecto mariposa, presente cuando en medio del caos pequeñas causas provocan grandes efectos.

En todo caso las teorías de la complejidad no son nuevas, ya que parten de la mecánica cuántica y la teoría de la relatividad, surgidas en franco antagonismo con la física clásica, de 
corte mecánico. Dichos modelos del mundo físico, al ser en su momento inéditos, dotaron a la percepción de lo real de mayor incertidumbre, al aceptar que los fenómenos no dependen de una sola causa, sino que son multifactoriales. Esto de alguna manera ha facultado su empleo dentro de las ciencias sociales, humanas y de la conducta, donde el paradigma tradicional de la física no ha podido expresarse plenamente, severamente cuestionado por quienes aseguran no pertenece a este rubro argumentativo. Los fenómenos sociales son los mejores beneficiarios de estos postulados, ya que desde hace décadas se sostiene su complejidad, sin que se haya acuñado una teoría -o teorías- que les respalden. Maldonado (2011) al señalar el discurso pronunciado por Ilya Prigogine al recibir el premio nobel en 1977, refiriéndose al postulado del no-equilibrio como el origen del orden, cita lo siguiente:

Es interesante el que la bifurcación introduzca en un sentido la 'historia' en la física... De este modo, introducimos en la física y la química un elemento 'histórico', el cual hasta ahora parecía reservado tan sólo a las ciencias que tratan con fenómenos biológicos, sociales y culturales. (p.25).

En conceptos de Maldonado, todo parece indicar que las diferencias entre las ciencias duras y blandas desaparecen, $\mathrm{o}$ al menos se disipan en sus discrepancias, conciliación que abre paso a una nueva síntesis del conocimiento.

En atención a estas premisas, se busca contribuir al esclarecimiento de los fundamentos que deben contemplarse al plantear y delimitar protocolos de investigación sustentados desde la complejidad, particularmente para el nivel superior y de posgrado, donde existe una creciente tendencia a orientar sus tesis de maestría y doctorado hacia esta vertiente de entendimiento.

\section{El fenómeno objeto de estudio desde la complejidad}

Si en la ciencia tradicional los fenómenos a estudiar son concebidos como relaciones entre variables que de alguna forma se hayan asociadas, en la teoría de la complejidad el principio atiende a las estructuras, sus componentes y las relaciones entre ellos. Luhmann (1990, 1991, 1996, 1997, 1998) considera que el sistema no es algo dado y listo para ser observado o intervenido, por lo que resulta interesante saber cómo se construye el sistema a partir de sus elementos y relaciones. ¿Qué es lo que hace posible un sistema? ¿Qué relaciona a sus elementos y cómo se organizan? ¿Qué limitantes enfrenta este proceso? No es ahora interesante la cantidad de elementos que lo integran, incluso el número de relaciones que establecen dichos elementos. Se habla de una complejidad sistémica que depende no tanto de las relaciones que se pueden establecer dentro de un sistema con base en su estructura, sino particularmente de la selectividad de dichas relaciones. Aquí reside la mayor o menor complejidad. El aumento en la selectividad de las relaciones depende del tamaño de la estructura del sistema, sin importar la relación directa entre tamaño de la estructura y número de relaciones -lo cual sería una relación lineal-. Desde la selectividad, las relaciones se crean y destruyen, por ello la complejidad se fundamenta en las relaciones que se establecen, las cuales surgen entonces por dicha selección. 
Sobre este particular se destaca lo expresado por Gómez (2010), quien considera que los postulados de Luhmann son extrapolaciones de las investigaciones sobre la autopoiesis, realizadas por Maturana \& Varela (1972).

El mismo Luhmann habla de dos tipos de complejidad: operacional y observacional. La primera distingue entre elementos y relaciones, es decir, a mayor composición de elementos en la estructura del sistema mayor dificultad para que sus elementos se relacionen; la segunda está basada en la observación, la cual se complica cuando el propio sistema selecciona las relaciones que establecerá, lo que provoca mayor complejidad, al no saber, incluso basándose en observaciones previas, qué relaciones se instaurarán después. ¿Qué interpretar entonces? ¿Bajo qué contextos, entornos o mundos se dan estas interpretaciones? Asumiéndose que estos escenarios son muchas veces más complejos que el propio sistema, ya que si el entorno es complejo sus sistemas lo serán. Sistemas que tienden a evolucionar -no a cambiar- si los demás sistemas del contexto mantienen su selectividad -no se mueven al azar-, donde el factor tiempo es relevante. Cuando se manifiesta la selección de una relación entre elementos dentro de la estructura se reduce su complejidad, ya sea se estabilice, se detenga o cambie. Principio de comunicación que se basa en el precepto de la complejidad y la contingencia que resulta de esperar una relación y generarse otra. He aquí nuevos campos para el estudio de los fenómenos sociales.

Sobre esta base los fenómenos sociales se sustentan en la complejidad del sistema, desde donde se pueden llegar a estudiar y comprender. Como ejemplos se tiene: 1) el papel de los impulsos positivos y negativos de las personas en torno a la comunicación; 2) la confianza como factor reductor de la complejidad; 3) el tiempo y su papel en la selección de las relaciones; 4) la estabilidad y mantenimiento de la autonomía del sistema; 5) la reducción de la complejidad a partir de la selección de las relaciones dentro de un sistema en un entorno específico; 6) tipo de selección y características de sus contingencias; y 7) el enriquecimiento de la teoría como proceso autopoiético a partir de la comunicación.

En otro cauce de ideas se encuentra lo postulado por Edgar Morin, quien apuesta a un abordaje no lineal de la realidad, donde un sistema complejo no puede ser parcializado, es decir analizado, lo que convierte la predicción en una dificultad en virtud de la autoorganización que lleva a relaciones de orden no predecibles. En sus conceptos se trata de "...concebir el sistema como unidad global organizada de interrelaciones entre elementos, acciones o individuos" (Morin, 1993, pp.123-124).

Para Morin (1994) un sistema complejo es una unidad global que puede manifestar propiedades inexplicables a partir de sus componentes. Esto se debe a que el todo expresa propiedades que no tienen significado a la luz de la dinámica de sus componentes; situadas como emergentes y colectivas, estas propiedades dan lugar a infinitas interacciones interrelaciones- no lineales. Es la interacción de las partes lo que define la complejidad del sistema. La idea de un sistema jerarquizado está vigente, integrado por subsistemas que se incluyen a sí mismos -bucles-, configuradores de la realidad; sistemas que coevolucionan con su entorno. Aquí yacen muchos de los principios de la Teoría de la Información de 
Claude E. Shannon (1998) y Warren Weaver (1948), la Cibernética de Norbert Wiener (1986), la Teoría de Sistemas de Ludwig von Bertalanffy, y el concepto de auto-organización de William Ross Ashby (2012), fundamentos a los que Morin afirma adherirse teóricamente.

Desde luego coexisten otras teorías que aportan fundamentos, tales como la geometría fractal (Mandelbrot, 1987), la teoría de los autómatas celulares (Von Neumann, 1966), la termodinámica de los procesos irreversibles (Prigogine \& Nicolis, 1987), la teoría de la autopoiesis (Maturana \& Varela, 1972) y la teoría de las catástrofes (Thom, 1974, 1988).

Su mayor respaldo sin duda se encuentra en la Teoría de Sistemas, exaltada por Morin (1994, pp. 41-42) como el eje articulador de sus conceptos. En sus palabras: "...toda realidad conocida, desde el átomo hasta la galaxia, pasando por la molécula, la célula, el organismo y la sociedad, puede ser concebido como sistema, es decir, como asociación combinatoria de elementos diferentes". Se establece así la idea de sistemas abiertos que se auto-organizan.

Soportada por el principio de equilibrio - relación existente entre creación y destrucción de las relaciones presentes en el sistema-, la propuesta de la complejidad supera el sentido original de la Teoría de Sistemas, donde la entropía es controlada mediante una neguentropía o entropía negativa -retroalimentación negativa, en la que el sistema exporta entropía para conservar su entropía baja-, particularmente frente a amenazas externas. El sistema busca subsistir estabilizándose frente a una situación caótica -de máxima entropía-. El caos se mantiene así en límites tolerables por el sistema. En los sistemas cerrados la tendencia es al caos y al desorden. La Teoría de Sistemas se entiende entonces como "... un juego activo de interacciones, retroacciones, emergencias, constreñimientos; allí los antagonismos entre las partes, entre las partes y el todo, entre lo emergente y lo sumergido, entre lo estructural y lo fenoménico, se ponen en movimiento" (Morin, 1993, p.179).

Bajo sus premisas, los elementos que integran el sistema deben definirse a partir de sus caracteres originales, las interrelaciones en que participan y la perspectiva que ofrece su organización, entendida como un todo. En términos concretos, dicha organización se define con relación a sus elementos y sus interrelaciones.

Morin, con base en los conceptos de Bateson (1972), destaca el papel de la comunicación, al asumir que esta media todas las experiencias y expresiones humanas, donde trabajo, lenguaje y pensamiento construyen la representación simbólica de la realidad; asumiéndose con ello deudor de los preceptos de Lev S. Vigotsky y Jean Piaget. Barberousse (2008) sintetiza el pensamiento complejo de Morin en tres aspectos básicos:

...primero la noción de círculo de las ciencias que fue asumida por Edgar Morin como círculo epistemológico mediante la integración de la idea de búsqueda inter y transdisciplinaria; segundo, la noción de sujeto epistémico auto-regulado para Piaget que se adapta al medio, que se transformó en la idea de sujeto incorporado a su contexto en una dialógica ecologizante de autoconformación sujeto-contexto $y$, tercero, la idea piagetiana de auto-regulación que se integró a la propuesta de un principio auto-eco-organizador en el cual el sujeto observador no puede concebirse aislado del objeto estudiado sino en y por su relación co-fundante con su 
objeto, con su entorno en el marco de un pensamiento contextualizante y ecologizante. (p. 111).

Ante la pregunta básica que plantea ¿Qué es la complejidad? el propio Morin (1994) aclara con puntualidad:

A primera vista la complejidad es un tejido (complexus: lo que está tejido en conjunto) de constituyentes heterogéneos inseparablemente asociados: presenta la paradoja de lo uno y lo múltiple. Al mirar con más atención, la complejidad es, efectivamente, el tejido de eventos, acciones, interacciones, retroacciones, determinaciones, azares, que constituyen nuestro mundo fenoménico. Así es que la complejidad se presenta con los rasgos inquietantes de lo enredado, de lo inextricable, del desorden, la ambigüedad, la incertidumbre... De allí la necesidad, para el conocimiento, de poner orden en los fenómenos rechazando el desorden, de descartar lo incierto, es decir, de seleccionar los elementos de orden y de certidumbre, de quitar ambigüedad, clarificar, distinguir, jerarquizar... (p. 32).

Introduce el concepto de complejidad antroposocial -Ser bioantropo: socio/cultural/histórico-, donde la vida es vista como fenómeno auto-organizado, de extraordinaria complejidad, que produce autonomía. A su vez específica que:

...el pensamiento complejo aspira al conocimiento multidimensional. Pero sabe, desde el comienzo, que el conocimiento completo es imposible: uno de los axiomas de la complejidad es la imposibilidad, incluso teórica, de una omnisciencia. Hace suya la frase de Adorno «la totalidad es la no-verdad». Implica el reconocimiento de un principio de incompletud y de incertidumbre. Pero implica también, por principio, el reconocimiento de los lazos entre las entidades que nuestro pensamiento debe necesariamente distinguir, pero no aislar, entre sí. (Morin, 1994, p.22).

Resulta entonces que la complejidad vista por Morin se sitúa en una eterna tensión entre el saber no parcelado, no reducido, y la incertidumbre que se deriva de reconocer que todo conocimiento es incompleto e inacabado.

Es precisamente esta inseguridad lo que mejor explica y a su vez respalda al pensamiento fraccionario; cosmovisión que llevó a simplificar la realidad, y con ello al pensamiento que la hace posible. Se trata efectivamente de un problema de organización del conocimiento. En un intento por reducir y abstraer aquello que se dice real, donde el sujeto cognoscente y el objeto cognoscible fueron separados, con el propósito de hacer claro aquello que se afirma observar.

En medio de estas posturas epistémicas es justo reconocer que la visión cartesiana parte también de aceptar la complejidad de lo real, solo que su forma de abordarla es distinta a la propuesta desde la complejidad. De ahí que el problema sea metodológico y no precisamente epistémico, aunque no deben desestimarse las diferencias que se señalan desde la naturaleza de los fenómenos: mientras un paradigma afirma poder explicar el todo a partir del estudio de sus partes, el otro espera encontrar en el estudio del todo la comprensión de sus partes. El primero reconoce el caos y por ello trata de encontrar un orden en las cosas, desarticulándolas y uniéndolas nuevamente bajo una jerarquía específica, que deriva de la aplicación de principios lógicos y legaliformes; el segundo acepta el caos y busca su entendimiento, asumiéndolo como una complejidad en la que los lazos 
establecidos entre sus partes definen al estudio; vínculos que, como ya se señaló, deben distinguirse, pero no aislarse.

Se asume entonces que el mundo fenoménico de la sociedad y su cultura es un fino tejido de eventos, acciones, interacciones, retroacciones, determinaciones (Zapata, 2005, p.41), e incluso azares, que se expresan en valores, rituales, representaciones simbólicas, creencias, saberes comunes y emociones, donde el caos como complejidad es la constante. Visto de esta forma todo hecho educativo está cargado de complejidad, convirtiéndose en un fenómeno factible de ser estudiado.

Pero el estudio de este tipo de fenómenos precisa de un sujeto-investigador que debe cubrir ciertas características ya identificadas líneas atrás. Se trata de un ser epistémico autoregulado, con una visión inter o transdisciplinaria, contextualizado y cofundador de la realidad. De aquí que se hable de pensamiento complejo, propio de los postulados de Morin. Condiciones sine qua non para abordar la complejidad.

Finalmente se trata de insistir en el binomio sujeto/objeto -lo cual no implica una novedad-, ahora vislumbrado como un sujeto que se asume parte de lo observado; estado procedente del juego activo de interacciones e interrelaciones que se dan dentro de una realidad, admitida como un sistema abierto.

Destacable en la obra de Morin es el múltiple referente que toma la realidad desde la complejidad, a la que Leyva-Rodríguez (2009, p. 7) interpreta al señalar que "todo conocimiento posee entonces componentes biológicos, culturales, cerebrales, sociales e históricos, del mismo modo que la idea más simple precisa de una complejidad bioantropológica y una hiper-complejidad sociocultural".

Rolando García, una de las voces más autorizadas sobre este tema en América Latina, establece que, al tratarse de problemas ambientales, es indispensable considerar que estos:

...corresponden a problemáticas complejas, donde están involucrados el medio físico-biológico, la producción, la tecnología, la organización social, la economía. Tales situaciones se caracterizan por la confluencia de múltiples procesos cuyas interrelaciones constituyen la estructura de un sistema que funciona como una totalidad organizada, a la cual hemos denominado sistema complejo... (García, 2011, p.66).

Se establece entonces la importancia de las interrelaciones dentro de la estructura, manifestadas a partir de múltiples procesos, que de acuerdo con García (2011) se dan al menos en dos niveles de complejidad -macro y micro-. Sin embargo, de acuerdo con sus conceptos, la complejidad presente en un sistema está dado por la heterogeneidad de los elementos que integran la estructura, interpretados como constituyentes de los subsistemas. Pero aún más: lo relevante es la interdefinibilidad y mutua dependencia de las funciones que se dan entre dichos elementos dentro del sistema total. Se destaca la forma en que García (2011) señala que los niveles micro -problemáticas específicas o situadas- son dominio de las diversas ramas de la ciencia -química, física, biología, etc.-, identificables como campos de 
interés multidisciplinarios, no necesariamente interdisciplinarios. Aclara atinadamente que por esta razón los sistemas complejos -macrosistemas- no pueden ser abordados sectorialmente, tal como ocurre con los microsistemas.

Son tal las implicaciones de una intervención -modificación- dentro de un sistema complejo que todas las relaciones dentro del sistema se verán afectadas, lo que en presencia de una baja resiliencia llevaría a una reorganización total.

La resiliencia señala las condiciones bajo las cuales un sistema complejo, apartado del equilibrio, se encuentra sometido a inestabilidades que pueden transformarlo, al grado de manifestar una nueva pauta de comportamiento. Sobre esta base es necesario determinar qué tanto este sistema complejo puede soportar los cambios a los que se ve sujeto, sin perder sus propiedades funcionales y modificar su estructura. Esto involucra su capacidad de auto-organizarse, para lo que el sistema debe desarrollar la habilidad de aprender y adaptarse.

Paradójicamente la existencia de una baja resiliencia puede llegar a ser una circunstancia deseada. Un ejemplo puede ser la introducción de un sistema de gestión de la calidad dentro de un nivel educativo, o el cambio de modelo educativo dentro de un sistema nacional. En términos de políticas educativas públicas esta condición es comúnmente el propósito de las intervenciones: lograr profundas modificaciones en las relaciones de los elementos que conforman la estructura del sistema, que lleven a modificar tanto a los elementos como a sus relaciones. La meta es modificar el sistema para generar una nueva estructura. Muchas propuestas de investigación parten del estudio de estos escenarios.

Con ello se recrea lo que García (2011) llama el juego dialéctico: al cambiar los elementos cambia la funcionalidad de la totalidad del sistema, al cambiar la funcionalidad cambia la reorganización de los elementos. Se trata de una doble direccionalidad de los procesos. Aquí radica la dificultad al estudiar la dinámica de los sistemas complejos. El modelo tradicional cartesiano afronta esto desde las ciencias conocidas, en un enfoque multi o pluridisciplinario; el pensamiento complejo debe hacerlo desde la interdisciplina. La manera de realizarlo es clara: "...no partimos de una definición de 'interdisciplina' in-abstracto, que luego aplicamos a ese objeto de estudio particular que es un sistema complejo. Por el contrario, definimos primero el objeto de estudio, y luego nos planteamos la manera de estudiarlo" (García, 2011, p. 67).

El autor enfatiza en la necesidad de acudir a la metodología interdisciplinaria para atender de manera adecuada estos fenómenos, entendidos como sistemas complejos; sin embargo, existe cierta ambigüedad en sus planteamientos, al señalar que: "En este contexto, metodología 'adecuada' significa que debe servir como instrumento de análisis de los procesos que tienen lugar en un sistema complejo y que explican su comportamiento y evolución como totalidad organizada" (García, 2011, p. 67). Obsérvese cómo se acude al análisis para hacer operable dicha metodología, lo que remite nuevamente al tradicional sistema fraccionario. Es necesario entender entre líneas que dicho análisis toma una 
acepción diferente, para este caso entendido como el ejercicio de pensamiento que estudia las relaciones que pueden establecer ciertos elementos al configurar una estructura, entendidos como subsistemas.

Sobre la base de sus postulados se pueden identificar las siguientes especificaciones:

1. No cualquier investigación es interdisciplinaria. Un sociólogo puede estar interesado en estudiar las percepciones sociales acerca de la ciencia y tecnología de un grupo de docentesinvestigadores en una institución educativa, para lo cual recurre a enfoques psicológicos y antropológicos, apoyándose en técnicas variadas. Esto califica como un estudio multidisciplinario, pero no interdisciplinario.

2. La complejidad de un sistema, por ejemplo el de un cuerpo académico, no radica en la heterogeneidad de sus elementos, es decir, en los perfiles profesionales o experiencia de sus integrantes, sino como ya se señaló, en las interrelaciones de sus elementos.

3. Los sistemas complejos exigen ser estudiados desde la interdisciplina y no al revés. No se buscan fenómenos de estudio o sistemas que estudiar bajo la interdisciplina, sino que son estos los que exigen un abordaje interdisciplinario. Un modelo educativo nacional, como sistema complejo está constituido por una propuesta curricular, didáctica, organizacional, pero a su vez es un proyecto político de Estado, integrado por funcionarios, directivos, maestros y personal de apoyo, cuyas interrelaciones explican la presencia de una estructura, reclamante de un abordaje interdisciplinario.

4. Los estudios desde la interdisciplina, orientados hacia los sistemas complejos, deben ser atendidos por investigadores que compartan paradigmas y modelos análogos, sin importar si poseen formaciones disciplinares distintas. Dicho en otros términos: el grupo de investigadores que estudia un sistema complejo debe ser también un sistema, no un conglomerado.

Adaptado de los conceptos de García (2011, p.68).

El esfuerzo de García (2011) por proyectar una metodología interdisciplinaria que atienda de manera distinta los sistemas complejos es por demás loable. Pese a ello, su insistencia por no abandonar los estudios especializados parece una contradicción, incluso la persistencia con la que defiende su empleo dentro de la propia investigación interdisciplinaria refrenda su apego al estatus quo de la investigación decimonónica. Al respecto indica:

...ni la condena a la "especialización excesiva" conduce, por oposición, a la interdisciplina, ni es posible prescindir de los especialistas aún en la investigación interdisciplinaria. Se trata de un problema mal formulado. No toda la investigación es interdisciplinaria, ni todo profesional necesita ocuparse de interdisciplina. (García, 2011, p.70).

Esta afirmación es clave: la realidad, aún entendida como sistema, en ocasiones puede y debe ser estudiada por especialistas en alguna disciplina, para ello es necesaria su presencia en el campo de la investigación.

La propuesta de Rolando García es formar generalistas, esto es, profesionistas que puedan ver el bosque y no solo el árbol. Aunque esto es más un deseo que una realidad. Incluso los grupos de investigación consolidados no llegan a constituirse en interdisciplinarios, cuando mucho en multidisciplinarios. Aunque en la práctica aparezcan como tales, invocar el término no lo hace posible. Como el propio Rolando García (2011, p. 71) establece: "La interdisciplinariedad no emerge espontáneamente poniendo juntos a varios especialistas. Con esta 'solución' el problema queda intacto". 
Finalmente, la mejor explicación sobre lo que significa un sistema complejo es la que establece que: "El conjunto de sus relaciones constituye la estructura, que da al sistema la forma de organización que le hace funcionar como una totalidad. De aquí el nombre de 'sistema'" (García, 2011, p.74). Con base en ello ¿Qué se puede investigar dentro de un sistema social y educativo? Desde luego sus propiedades, su vulnerabilidad, su resiliencia, su dinámica de evolución, e incluso las bases conceptuales con las que se intenta comprender la interdisciplina como método de estudio. Aunque García (2011) propone empezar todo estudio integral con un diagnóstico del funcionamiento del sistema.

Es de llamar la atención que Rolando García exponga, dentro de las etapas o fases que debe incluir el diagnóstico, la misma ruta que regularmente se sigue en los procesos de pensamiento deductivo, propios de la ciencia tradicional, tales como la problematización, la construcción de un marco teórico referencial, identificación de elementos o relaciones entre ellos, planteamiento de hipótesis y su verificación para aceptar o refutarlas, con pequeñas adiciones alusivas a la identificación de las problemáticas identificadas dentro de los subsistemas, pero reiterando el empleo de las investigaciones disciplinarias -especializadas-, que no permiten identificar una visión distinta desde la complejidad (véase García, 2011, p. 81). Esto no es privativo de este autor, ya que Hayles (1990), citado por Cárdenas \& Rivera (2004), indican al respecto que el caos o la complejidad:

Es ciencia normal. Sigue el mismo procedimiento que cualquier otra disciplina científica para acreditar a sus miembros, diseñar protocolos de investigación y evaluar resultados. (...) Los criterios y los procedimientos de la ciencia normal no han cambiado; lo que ha cambiado es el fundamento epistemológico sobre el que se basa, sobre todo en la cultura contemporánea. Cuando se desestabiliza una dicotomía tan decisiva para el pensamiento occidental como la de orden/desorden, no es exagerado decir que se ha abierto una importante falla geológica en la episteme. (Cárdenas \& Rivera, 2004, pp. 1336-137).

Sobre las premisas de este autor, lo primero que hay que hacer es eliminar la diferenciación entre ciencias biológicas, químico-matemáticas y sociales y de la conducta, a la cual es común referirse. Aunado a ello, se debe subrayar que las teorías de la complejidad anidan en los sistemas complejos, entendidos en sentido amplio como sistemas dinámicos, sistemas no lineales, sistemas adaptativos, que incluyen la teoría del caos y los fractales (Castillo-Villanueva \& Velázquez-Torres, 2015). Como se puede apreciar:

...no existe en la actualidad, una teoría unificada de la complejidad, que sintetice y sistematice de modo explícito los aspectos fundamentales de las distintas y variadas teorías, métodos y algoritmos de complejidad elaborados en el marco de ciencias y disciplinas disímiles. (Rodríguez-Zoya \& Aguirre, 2011, p.3).

Maldonado (2011), lo expresa de forma más amplia:

Es importante atender al plural en la expresión "ciencia de la complejidad". En contraste con la ciencia clásica, el estudio de la complejidad tanto da lugar a, como convoca, lenguajes, teorías, métodos, lógicas y aproximaciones diversas, todas de orden inter y transdisciplinario, por lo cual se las designa en plural. Pues bien, históricamente hablando, la primera de las ciencias de la complejidad es la termodinámica del no-equilibrio, forma más genérica de referirse a, y estudiar, la 
complejidad, esto es, aquellos procesos, fenómenos y comportamientos caracterizados por fluctuaciones, inestabilidades, autoorganización y emergencias, en fin, desequilibrios o equilibrios dinámicos, permitiendo así avanzar con respecto a la polisemia de "complejidad", es decir, a la ausencia de una definición única de "complejidad". (p. 23).

En conceptos de Warren Weaver (1948), es indispensable pensar en tres momentos en la historia de la ciencia, asociados a la forma de ver y abordar sus problemáticas: problemas de simplicidad, problemas de la complejidad desorganizada y problemas de la complejidad organizada. La primera basada en la física newtoniana -modelos mecánicos-; la segunda apoyada por la estadística avanzada, multivariada o multifactorial; la tercera sostenida por la idea, no de múltiples variables interaccionando, sino de relaciones que se establecen entre ellas, conformando un todo, relaciones estudiadas mediante modelos sistémicos. En conceptos de Morin: complicación en los dos primeros y complejidad en el último. Como ya se estipuló, Maldonado (2011, p. 25) señala como hecho histórico que la "...termodinámica del no-equilibrio es la primera de las ciencias de la complejidad", lo que sitúa a Ilya Prigogine como el fundador de este nuevo paradigma.

Los tres autores aquí presentados exhiben la doble postura con la cual actualmente se intenta proyectar el estudio de la complejidad. Edgar Morin desde el Pensamiento Complejo complejidad en términos de organizaciones, es decir, totalidades organizadas por elementos heterogéneos en interacción-; Niklas Luhmann y Rolando García desde las Ciencias de la Complejidad -complejidad de un sistema o totalidad organizada-. Ambas vertientes distinguibles y vinculadas a través de la Teoría de Sistemas de Bertalanffy.

En un ejercicio de reflexión más amplio se puede afirmar que Edgar Morin denomina complejidad restringida a las Ciencias de la Complejidad -postura anglosajona, con soporte técnico/metodológico-, mientras que el pensamiento complejo atiende una visión franco/latina, más epistémica y transdisciplinaria -complejidad general-, con una filosofía ético-política, planetaria y apegada a la naturaleza. Por otra parte, Maldonado (2011) distingue en Morin una corriente de la «complejidad como método», identificándolo también como el padre del Pensamiento Complejo. Sobre esta base Rodríguez-Zoya \& Aguirre (2011, p. 9) lo descalifican de alguna manera al indicar que se trata de las ideas de un solo autor, aunque también establecen la duda sobre los usos del saber derivado de las Ciencias de la Complejidad al preguntar: "qué intereses rigen la producción de conocimiento en ciencias de la complejidad, y a quiénes benefician los saberes por ellas elaborados". El propio Rolando García expone una crítica muy severa sobre el pensamiento de Morín, de quien puntualiza: "Edgar Morin contribuyó a demoler las bases del racionalismo tradicional que había penetrado tan profundamente en el sistema educativo francés (fundamentado en el Discurso del Método de Descartes). Sin embargo, su crítica no ofrece una formulación precisa de los problemas que enuncia (problemas que el cartesianismo dejó pendientes y que corresponden al campo de la teoría del conocimiento) como para conducir a una metodología de trabajo aplicable a las situaciones concretas que él considera como 'complejas'." (García, 2006, p. 21). Lo que no le perdonan a Morin es que su tan reconocido método no muestre cómo obtener evidencias empíricas. Se trata de un camino para llegar a un fin, pero un fin teórico-epistémico, no técnico. En su defensa Morin argumentará que es 
necesaria una epistemología sobre el sujeto, no sobre los contenidos de sus experiencias. Un sujeto así, organiza y construye activamente la realidad, y con ello el conocimiento -sujeto observador/conceptualizador-. La complejidad para él no es una respuesta, sino el reconocimiento de las incertidumbres y contradicciones; en sus palabras se trata de un desafío al pensamiento.

\section{El trabajo del investigador social}

Los fundamentos de la complejidad remiten a una disyuntiva para el investigador: ¿Los investigadores de la complejidad deben atender los fenómenos sociales como un nuevo campo de su desempeño científico? O bien ¿Los científicos sociales deben acudir a las ciencias de la complejidad para abordar conceptualmente sus investigaciones? Incluso: ¿Los investigadores sociales y de la conducta deben, como ya se señaló, poseer un pensamiento complejo como requisito para acceder a las ciencias de la complejidad?

La ventaja observable estriba precisamente en el reconocimiento generalizado que los estudiosos de las ciencias sociales y de la conducta le confieren a los procesos formativos humanos, culturalmente significativos para ellos desde el siglo XX, donde personajes como Bronfenbrenner $(1974,1977,1979)$ y su Teoría Ecológica de la Educación pusieron el énfasis en la estructura ambiental que define y explica un sistema desde distintos niveles, más o menos cercanos al individuo -familia, escuela, vecinos, amigos, la etapa histórica en que se vive, los eventos globales que le afectan, entre otros-, que va del microsistema al globosistema; tránsito que incluye el mesosistema, exosistema, macrosistema y el cronosistema. El propio Gadotti (2002) apoya esta visión holista -centrada en el sentido de la totalidad- al manifestar la necesidad de una Pedagogía de la Tierra, que comprenda la Ecopedagogía y la Educación Sustentable, modelos que aspiran a transformarse en paradigmas dominantes dentro del campo de la formación escolar formal, e incluso en la informal y no formal. Educación para la vida que de consolidarse significaría una nueva revolución científica en un sentido kuhniano.

¿Cuál es entonces el camino a seguir dentro de la investigación social, específicamente en la educación? De Jesús, Andrade, Martínez \& Méndez (2007) brindan pautas para encontrar una respuesta:

Educación y conocimiento, conocimiento y educación, son los dos pilares sobre los que la humanidad asienta sus procesos transformadores. Hábitos, valores, pautas culturales, prácticas, conductas, métodos, formas, maneras, contenidos, mitos, sueños, leyendas, ciencia, técnica, arte y cualquier forma del hacer humano se entrelaza con alguna manifestación de lo educativo desde la perspectiva de arte, ciencia o camino. (p.3).

Es este entrelazamiento lo que mejor representa la complejidad de lo social; la estructura conformada por el fino tejido que integra el sistema de relaciones entre los actores del hecho socioeducativo. Pero la relevancia no está en los rituales ni las prácticas socioculturales ahí 
consagradas, sino en las relaciones de que derivan y hacia las que conducen, particularmente en el sentido y la intención implícitos en cada hecho educativo.

El conocimiento identificado por De Jesús et al., (2007) tiene por naturaleza un fundamento epistémico plural, compuesto por saberes diversos, tan disímiles que llegan a conformar una ecología de los saberes (De Sousa-Santos, 2010) que desafía al conocimiento científico -de ascendencia lineal-, y lo sitúa frente a un obligado diálogo de saberes; descolonización del saber hegemónico equivalente a un des-pensar para poder pensar (De Sousa-Santos, 2010).

Como se observa se trata de posturas emanadas del pensamiento complejo que colisionan con el basamento epistémico de las Ciencias de la Complejidad, proclive a sostener la idea de un orden dentro del caos, predecible en tanto puede ser identificado presente incluso en la propuesta de Bronfenbrenner (1979)-. El mayor problema radica en lo inédito de cada evento sociocultural, que en la educación se traduce como una secuencia de hechos irrepetibles, que van más allá de sus protagonistas, para situarse en las interrelaciones que logran concertar, consciente o inconscientemente.

El pensamiento complejo, al ser concebido como una unidad global organizada de soporte moriniano-, sitúa su interés en el mundo fenoménico de la sociedad y su cultura, diverso y problemático. Pero una cosa es pensar a la educación como una complejidad y otra concebirla desde la complejidad.

Más allá de los debates en torno a lo complejo que resulta la educación desde las perspectivas tradicionales, actualmente se plantean nuevos retos que bien pueden ser entendidos y abordados desde el pensamiento complejo, a saber se tiene: 1) el agotamiento de la escuela como institución social; 2) el papel que juega la escuela en la consolidación de un modelo educativo globalizante impulsado por organismos internacionales; 3) la capacidad resiliente de las instituciones educativas frente a las perturbaciones provocados por las reformas educativas; 4) la pérdida del referente humanista en el proceso enseñanzaaprendizaje mediado por las tecnologías de la información y la comunicación; 5) la sustitución del tradicional tutelado de los padres por el ejercicio de tutoría de los maestros; 6) el reemplazo de los padres de familia por el de los empresarios en las decisiones curriculares; 7) el cambio de las didácticas usuales centradas en el maestro, por aquellas orientadas a partir de las necesidades del estudiante; 8) el empleo de aulas invertidas que convierten a la escuela en centros de evaluación y no de enseñanza; 9) el predominio de la calidad educativa sobre la educación de calidad en los proyectos de mejora continua institucional; 10) la tendencia cada vez más dominante hacia una educación basada en competencias, con la consabida pérdida del sentido universal de la educación. Muchas de estas situaciones problemáticas expuestas abiertamente por Freire $(1974,1990,1993)$ y Gramsci (1971, 1979, 1985). 
Los tres primeros puntos se significan por ser campos de atención de la pedagogía crítica, apoyada por Peter McLaren (1994) y Henry Giroux (2005).

Esto son solo algunos ejemplos de los campos problemáticos que pueden ser abordados como sistemas complejos, fenómenos que no reportan líneas divisorias claras y precisas, como figuras de demarcación conceptual y operativa que puedan ser precisadas por el intelecto disciplinar.

La clave está en averiguar acerca de los propósitos que subyacen a los diversos proyectos educativos, y no solo en identificar sus constantes y variables. Espejo (2010) lo enfatiza al preguntar:

¿Cuáles son las medidas y los compromisos que debemos tomar como sociedad para pensar en una educación compleja para las nuevas generaciones? La coherencia entre los medios y los fines es quizás una clave, la cual se inserta en un ciclo de recursividad que debemos tomar en cuenta. (p.12)

A diferencia de las ciencias físicas, donde el interés está centrado en lo que es, y no en lo que representa, en las ciencias sociales, humanas y de la conducta -que no por serlo dejan de ser físicas-, el meollo del asunto se aloja en los significados, en las percepciones y representaciones, en los simbolismos que rodean un hecho y lo transforman mientras lo interpretan.

Por ello hay quienes apuestan a la transdisciplina como vía para entender y atender la complejidad de lo social y educativo, en busca de generar una sana distancia con lo disciplinar, condición que no se logra con la interdisciplina.

Cargada de controversias, la transdisciplina no ha podido infiltrar con fuerza el pensamiento científico. Al respecto De la Herrán (2011, p. 3) enfatiza en la necesidad de poseer un enfoque disciplinar como requisito para alcanzar un enfoque transdisciplinar, e incluso descalifica a quienes destacan las bondades de este enfoque al señalar que "lo transdisciplinar 'sin lo' disciplinar o 'contra lo' disciplinar es pseudo, pura apariencia". Aunado a ello aporta una definición de transdisciplinariedad que no contribuye a dirimir el debate: "Si las disciplinas están compuestas de temas, y todos los temas son intrínsecamente transdisciplinares, deducimos que cualquier disciplina es íntimamente transdisciplinar." (De la Herrán, 2011, p.4). La premisa que sostiene esta conclusión no puede ampararse en la realidad, ya que es imposible afirmar que todos los temas son transdisciplinarios. En sus conceptos toda disciplina desemboca en la transdisciplina, por lo que esta es relativa.

Minimizar el campo o enfoque transdisciplinar no es una alternativa viable para posicionar a la interdisciplina, menos aún acotar la problemática de la investigación al pensar que esta "... se reduce, pues, una vez más, a una cuestión de actitudes de apertura y de sensibilidad investigadora" (De la Herrán, 2011, p.6). Se necesita en todo caso una mayor atención al campo teórico que le respalda, terreno donde aún no puede competir con la interdisciplina. 
Visto desde otro ángulo, la transdisciplina ofrece la posibilidad de vincular la complejidad con el diálogo de saberes. Pérez-Luna, Moya-Norys \& Curcu-Colón (2013) sostienen que "para establecer la relación entre educación y transdisciplina es necesario partir de un concepto ordenador que permita explicar las implicaciones pedagógicas del proceso de transversalidad como elemento base del diálogo de saberes" (p.16). Diálogo que según los autores remite al concepto de totalidad sostenido por trans-saberes -de carácter intersubjetivo-, admitidos como reconstrucciones mentales de una realidad compleja y multidimensional. Aquí se muestra la existencia de una estructura, cuyas relaciones pueden ser explicadas por la transversalidad señalada, generadora de un cruce de saberes que se encuentran y desencuentran transdisciplinariamente, dando forma al diálogo. Transversalidad y transdisciplinariedad como un binomio posible. Pérez-Luna et al., (2013) lo expresan así:

Lo transdisciplinario, desde una mirada educativa, representa una búsqueda que no se restringe a lo disciplinar, sino que concibe al saber y sus relaciones desde la idea de la totalidad como una manera de pensar lo real. Se produce una nueva lectura de los conceptos porque la realidad no es estática, sino que se despliega en su propio movimiento histórico. Es decir, el proceso educativo permite, a través del diálogo de saberes, un pensar transversal que a su vez implica la búsqueda en lo transdisciplinar. (p.16).

Diálogo de saberes que lleva a pensar transversalmente para obrar en lo transdisciplinario, y de ahí, reconstruir el diálogo. Circulo virtuoso que la transversalidad hace posible y que la transdisciplina admite al incorporar saberes diversificados, marginales, no escuchados, así como maneras distintas de concebir el conocimiento, identidades y experiencias (Pérez-Luna et al., 2013).

Esta condición apunta hacia dos campos fértiles: 1) uno disciplinario, en secuencia con el proceso evolutivo que lleva a resolver el problema de la linealidad y el conocimiento único disciplinar, en busca de superarlo con nuevas lógicas discursivas -Véase la obra de Enrique Leff para mayores detalles-; 2) el otro asociado directamente a la recuperación de nuevas voces que se expresen en saberes culturalmente diferenciados, donde destaca la obra de Juliana Merçon y colaboradores dentro del Instituto de Investigaciones en Educación. Universidad Veracruzana, Xalapa, México (Merçon, 2016; Merçon \& Nuñez, 2016; Merçon \& Sarmiento-Sánchez, 2016), interculturalidad que el propio Pérez-Luna et al., (2013) esclarece al puntualizar que:

...la ruptura con las lógicas disciplinarias es un imperativo en los actuales momentos, pues los conocimientos tienen que comunicarse, transversarse para una mejor comprensión, es decir la producción de una transformación recíproca de la organización disciplinar. En este sentido, se deben ir generando nuevos marcos de comprensión del mundo que habitamos y que nos habita, pues la nueva mirada transdisciplinaria debe resignificarse en un lenguaje polívoco, metafórico capaz de nombrar, no la realidad unívoca del discurso científico, sino la polifonía de la diversidad y la pluralidad. (p.18).

Al final resulta un sinsentido continuar con discordias bizantinas centradas en diferenciar estas dos vertientes. Lo disciplinario debe conciliarse con la interculturalidad, en tanto esta se asume consignataria del universo simbólico de las sociedades. Intersubjetividad que por 
naturaleza centra su razón de ser en la libertad para interpretar el mundo, para recrear el aprehender multidimensional en escenarios caóticos. En palabras de Pérez-Luna \& MoyaNorys (2008):

La investigación constituye la herramienta para desplegar el plano gnoseológico y abre la posibilidad de que el diálogo de saberes se corresponda con una búsqueda en lo transdisciplinario. En este sentido, la diversidad del pensar propicia espacios para la duda, la creatividad, la autonomía. El sujeto regresa a ser protagonista, se hace consciente de su relación con la realidad y deja de ser prisionero de los contenidos... (p.459).

Es así como el diálogo de saberes tiende a ser transdisciplinario, asumiéndose la complejidad como parte de su esencia, al aceptar el conocimiento como una construcción social que se proyecta como realidad aprehensible, siempre provisional; saber derivado de intensas búsquedas transversales generadoras del pensamiento complejo. He aquí el camino a seguir en el campo de la investigación dentro de las ciencias sociales, humanas y de conducta.

\section{CONCLUSIONES}

Uno de los mayores obstáculos para alcanzar el nivel de discernimiento que se reclama dentro de las ciencias sociales, humanas y de la conducta pasa necesariamente por entender que las lógicas tradicionales surgen como producto de acontecimientos históricos, de procesos sociales evolutivos, condicionados por factores políticos y económicos que impactaron fuertemente en la cultura, que derivaron en lo que Gómez \& Rubio-González (2017) denominan el saber «histórico-cultural». La visión fragmentaria y funcional no es por tanto producto de la falta de raciocinios, sino de la conveniencia de situar un pensamiento por encima de otro.

La tendencia a privilegiar un pensamiento simple, lineal, que explica con fines predictivos, se encuentra asociado a las conocidas disputas entre ideologías progresivas y religiosas, y desde luego a las nacientes industrias del siglo XVIII y XIX. La ruptura que propone PérezLuna et al., (2013) solo pudo ser viable como posibilidad hasta la segunda mitad del siglo XX, cuando el posmodernismo logró captar la atención intelectual ante la imposibilidad del paradigma experimental, empírico-analítico -heredero del modernismo del siglo XVII y XVIII, de seguir explicando la realidad en el universo. Pero ello no significa la inexistencia de la complejidad, la transversalidad y la transdisciplina hoy discutida; al contrario, permaneció latente hasta que la historia -y la condición humana- la puso en relieve. Basta recordar que el posmodernismo emerge como un movimiento cultural, artístico, filosófico y literario, que en occidente se posiciona como alternativa de expresión de lo que es humano.

De ello se desprende la idea de saberes marginados, latentes, que se entrecruzan para dar origen a reconstrucciones mentales promotoras del caos y la incertidumbre, escenarios ahora comunes, donde el pensamiento se crea y recrea para dar lugar a realidades alternativas. 
La lógica del error ya no morará más en la óptica del investigador. La ciencia y sus axiomas como principios legaliformes darán paso a severos cuestionamientos, al no poder explicar todo lo que en la realidad se observa. Incluso la predicción se volverá caótica: al final Dios si juega a los dados.

Sobre esta base es necesario orientar los debates lejos de la simple diferenciación entre ciencia de la complejidad y ciencias sociales, humanas y de la conducta, y su eventual conexión, alianza que a decir de Payares-Loyo (2011, p. 19) "sucede a través del diálogo, en el camino del estudio de la complejidad y el trabajo con complejidad".

Respecto a lo anterior, el que los físicos hayan tenido la oportunidad de verificar su existencia no significa que para las ciencias sociales y humanas sea así. La novedad es la forma en que las ciencias tradicionales conciben la complejidad, sobre todo su abordaje metodológico, desestimando la necesidad de una epistemología que la explique cabalmente, tal como la planteada por Morin, quien por cierto es denostado por aquellos que sostienen que su método no es verificable. Más de lo mismo.

El no aceptar que existen planos de la existencia física hace que las relaciones y los procesos sociales pretendan ser vistos desde las teorías que explican el caos, sin embargo, sus expresiones matemáticas siguen el mismo patrón ontológico propio de la mecánica, solo que ahora definida como cuántica. Se soslaya que el comportamiento humano es más que empiria, ya que lo fundamental del asunto estriba en la intención que yace en cada representación -individual o social- de su conducta. Las intenciones, los simbolismos, las creencias y los valores que mueven la compleja autonomía humana no deben ser encapsulados en silogismos, heurísticos y algoritmos, propios del pensamiento hegemónico. Campos como la antropología, sociología, psicología, y desde luego la filosofía y la educación, siempre lo han sabido.

En este tenor, la educación, como un ejemplo en particular, no necesita ser acreditada como ciencia desde la complejidad. La complexa estructura que integra cada sistema educativo es por demás notoria. Como afirma Arriaga (2017, p. 273), "no es pertinente hablar de la educación en abstracto, conviene más referirse a prácticas educativas; es decir, a actos humanos intencionales que se presentan como una realidad fenoménica". Los actores, sus circunstancias, las intenciones que subyacen a cada acto educativo, el valor que se les otorga, los lenguajes que la hacen comprensible, el sentido adquirido y el otorgado, la inmanencia del saber construido, la herencia social intangible expresada como legado; este es el terreno natural de la complejidad. Lo social siempre es complejo, porque la complejidad anida en lo social. Por estas razones es necesario coincidir con Rodríguez (2017), quien recomienda empezar por abordar la complejidad como "una visión filosófica de los saberes" (p.436), más que como un dominio alcanzado a partir de la adquisición estricta de conocimientos. 
Bajo estos sustentos se acepta que "...la educación forma parte de las ciencias sociales del no equilibrio" (Maldonado, 2014, p.4), donde "el pensamiento complejo, sale al paso no solamente de soluciones simples, sino que se opone a la mega especialización de los profesionales, ya que implica la renuncia a afrontar los problemas de manera integral" (Flores, 2011, p.27). Este no es un asunto menor, ya que la educación no busca solamente formar seres humanos que entiendan el mundo en que viven, sino además que sean capaces de transformarlo. Situación que requiere una visión sistémica y compleja, que no se puede alcanzar con la especialización a ultranza.

Si bien "...el locus de la educación en general -incluyendo pedagogía, filosofía de la educación, didáctica y demás- ha sido el de las ciencias sociales; más específicamente las aplicadas." (Maldonado, 2014, pp.8-9), es indispensable reconocer que el predominio de los modelos clásicos dentro de la educación -claramente disciplinarios y especializados-, se debe más a aspectos relacionados con el predominio de poder que con las propias inercias y dinámicas de trabajo emergidas dentro del campo sociocultural de la educación. Dicho de otra manera: las transformaciones en el proceso enseñanza-aprendizaje suelen no estar sujetas a los movimientos históricos de los sujetos en su calidad de actores sociales. En conceptos de Gómez (2010, p.195) "fraccionar o reducir el proceso representa poder." Por ello la complejidad no ha encontrado espacio en los entornos escolares para su consolidación como pensamiento dominante.

Para romper con este círculo intemperante se debe asumir la realidad social, humana y de la conducta como un proceso complejo, lo que obliga a contextualizar el pensamiento bajo propuestas epistémicas, teóricas y metodológicas que ayuden a entender qué es la complejidad y cómo opera, lo que abrirá un vasto campo para el desarrollo de enfoques y abordajes transdisciplinarios y holistas dentro de la educación, los cuales puedan orientar nuevas vetas de indagación dentro de la investigación, ahora desde la complejidad. Se trata no solo de saber acerca del mundo, sino de interactuar con él, con sus complejidades naturales y las inducidas, mediante perspectivas dialógicas y plurales, en busca del anhelado complex donde anida el diálogo de saberes, su gestión y negociación; configuraciones articuladas desde una competencia que deja de ser instrumental para concebirse como una aptitud propia del sujeto, que se abre ahora a un pensamiento que reconoce todas las dimensiones del ser humano.

En el campo de la educación este reto es significativo, pero afortunadamente las expectativas son promisorias. Lo primero que hay que dilucidar es si se debe pensar en la educación como una complejidad, o se debe identificar la complejidad -y sus múltiples facetas- dentro de la educación; o aún más: si es la complejidad una perspectiva teóricoconceptual que precisa de abordajes metodológicos que la hagan posible dentro de la educación. Como en todo campo complejo, es indispensable una nueva epistemología que recupere estas y otras reflexiones, que identifique nuevos problemas y brinde acceso a la sinéctica dentro del proceso creativo que conduce a su solución. Quienes se comprometan con esta tarea deben poseer las habilidades dialógicas necesarias, a fin de enfrentar las paradojas, contradicciones y antinomias presentes en la educación y en sus modelos clásicos 
de enseñanza-aprendizaje, en los mitos que alimentan la relación maestro-estudiante, en los antagonismos y las recursividades presentes en los nuevos enfoques y modelos, en su insistente ubicuidad, que únicamente puede ser comprendida si se revisa su ontogenia, pero también su filogenia, en busca de explicar los porqués y no solo los cómo de la educación.

\section{REFERENCIAS}

Arriaga, J. (2017). Sistema, autopoiesis y entropía en los desafíos educativos contemporáneos. Educación y Humanismo, 19 (33), 271-288. DOI: http://dx.doi.org/10.17081/eduhum.19.33.2644

Barberousse, P. (2008). Fundamentos teóricos del pensamiento complejo de Edgar Morin.

Revista Educare, 12 (2), 95-113. Recuperado de:

http://www.redalyc.org/pdf/1941/194114586009.pdf

Bateson, G. (1972). Pasos hacia una ecología de la mente. Buenos Aires: Editorial LohléLumen.

Bronfenbrenner, U. (1974). Development research, public policy, and the ecology of childhood. Child Development, (45), 1-5. DOI: http://dx.doi.org/10.2307 / 1127743

Bronfenbrenner, U. (1977). Lewinian space and ecological substance. Journal of Social, (3), 199-213. DOI: http://dx.doi.org/10.1111/j.1540-4560.1977.tb02533.x

Bronfenbrenner, U. (1979). The ecology of human development. Cambridge: Harvard University Press.

Cárdenas, R \& Rivera, R. (2004). La teoría de la complejidad y su influencia en la escuela. Revista de Teoría y Didáctica de las Ciencias Sociales, (9), 131-141. Recuperado de: http://www.redalyc.org/pdf/652/65200908.pdf 
Castillo-Villanueva, L. \& Velázquez-Torres, D. (2015). Sistemas complejos adaptativos, sistemas socio- ecológicos y resiliencia. Quivera, 17 (2), 11-32. Recuperado de: http://www.redalyc.org/pdf/401/40143424002.pdf

De Jesús, M., Andrade R., Martínez, M. \& Méndez, R. (2007). Re-pensando la Educación desde la Complejidad. Polis, Revista Latinoamericana, 6 (16), 1-15. Recuperado de: http://polis.revues.org/4581

De la Herrán, A. (2011). Complejidad y Transdisciplinariedad. Revista Educação Skepsis, Formação Profissional, (Contextos de la formación profesional), 1 (2), 294320. Recuperado

de:

https://www.uam.es/personal_pdi/fprofesorado/agustind/textos/completrans.pdf

De Sousa-Santos, B. (2010). Descolonizar el saber, reinventar el poder. Uruguay: Ediciones Trilce.

Espejo, R. (2010). Algunos aspectos de la educación compleja. Polis, Revista Latinoamericana, 9 (25), 119-135. DOI: http://dx.doi.org/10.4067/S071865682010000100007.

Flores, J. (2011). Complejidad y educación. Revista Diálogos, 5 (7), p. 23-34. Recuperado de: http://www.redicces.org.sv/jspui/bitstream/10972/2048/1/2.\%20Complejidad\%20y\% 20educacion.pdf

Freire, P. (1974). Educación para el cambio social. Buenos Aires: Tierra Nueva.

Freire, P. (1990). La naturaleza política de la educación. Cultura, poder y liberación. Barcelona: Ediciones Paidós.

Freire, P. (1993). Pedagogía de la esperanza: un encuentro con pedagogía del oprimido. México: Siglo XXI.

Gadotti, M. (2002). Pedagogía de la tierra. México: Siglo XXI. 
García, R. (2006). Sistemas complejos. Conceptos, método y fundamentación epistemológica de la investigación interdisciplinaria. Barcelona: Gedisa.

García, R. (2011). Interdisciplinariedad y sistemas complejos. ReLMeCS. Revista Latinoamericana de Metodología de las Ciencias Sociales, 1 (1), 66-101. Recuperado de:

http://contenidosabiertos.academica.mx/jspui/bitstream/987654321/504/1/interdiscip linariedad_y_sistemas_complejos.pdf

Giroux, H. (2005). Take Back Higher Education. Nueva York: Palgrave.

Gómez, T. (2010). El nuevo paradigma de la complejidad y la educación: una mirada histórica. Polis, Revista de la Universidad Bolivariana, 9(25), 183-198. Recuperado de: https://scielo.conicyt.cl/pdf/polis/v9n25/art10.pdf

Gómez, T \& Rubio-González, J. (2017). Complejidad-educación: un aporte para las generaciones futuras. Revista Educación y Humanismo, 19(33), 409-424.DOI: http://dx.doi.org/10.17081/eduhum.19.33.2653

González, J. (2009). La teoría de la complejidad. Dyna, 76 (157), 243-245. Recuperado de: http://www.redalyc.org/pdf/496/49611942024.pdf

Gramsci, A. (1971). SelectionsfromthePrison Notebooks. London: Lawrence\&Wishart.

Gramsci, A. (1979). LettersfromPrisonby Antonio Gramsci. London: Quartet.

Gramsci, A. (1985). Selectionsfromthe Cultural Writings. London: Lawrence \&Wishart.

Leyva-Rodríguez, J. (2009). Los presupuestos teóricos de la epistemología compleja. Revista de filosofía $A$ Parte Rei, 61, 1-12. Recuperado de: http://serbal.pntic.mec.es/AParteRei/leyva61.pdf 
Luhmann, N. (1990). Sociedad y sistema: la ambición de la teoría. España: Ediciones Paidós Ibérica, S. A.

Luhmann, N. (1991). Sistemas sociales. México: Ed. Universidad Iberoamericana/Alianza.

Luhmann, N. (1996). Teoría de la sociedad y pedagogía. España: Ed. Paidós Educador.

Luhmann, N. (1997). Organización y decisión. Autopoiesis, acción y entendimiento comunicativo. España: Ed. Anthropos. Universidad Iberoamericana.

Luhmann, N. (1998). Complejidad y modernidad. De la unidad a la diferencia. España: Editorial Trotta.

Maldonado, C. (2011). Termodinámica y complejidad. Una introducción para las ciencias sociales y humanas. Bogotá: Ediciones Desde Abajo.

Maldonado, C. (2014) ¿Qué es eso de pedagogía y educación en complejidad? Intersticios Sociales. El Colegio de Jalisco, 7, 1-23. Recuperado de: http://www.scielo.org.mx/pdf/ins/n7/n7a2.pdf

Mandelbrot, B. (1987). Geometría fractal de la naturaleza. Barcelona: Tusquets Editores S.A.

Maturana, H \& Varela, F. (1972). De máquinas y seres vivos. Santiago: Editorial Universitaria.

McLaren, P. (1994). Pedagogía Crítica y Cultura depredadora. Buenos Aires, Argentina: AIQUE.

Merçon, J. (2016). Construyendo nuevos posibles a partir de la articulación entre resiliencia, aprendizaje social y sistema escolar. Educação. 39 (1), 105-112. DOI: http://dx.doi.org/10.15448/1981-2582.2016.1 
Merçon, J \& Núñez, C. (2016). Siete desafíos de la autogestión en la construcción de procesos de aprendizaje social y resiliencia comunitaria. En: Astudillo, J.; Villasante, T.R. (comp.). Participación social con metodologías alternativas desde el Sur. Quito: Abya-Yala.

Merçon, J \& Sarmiento-Sánchez, A. (2016). Co-creación de saberes, poderes, prácticas e identidades campesinas en Tlaxcala, México. LEISA Revista de Agroecología. 32 (1), 15-16. Recuperado de: http://www.leisaal.org/web/images/stories/revistapdf/vol32n1.pdf

Morin, E. (1993). El Método I: La naturaleza de la naturaleza. Madrid: Cátedra.

Morin, E. (1994). Introducción al pensamiento complejo. Barcelona: Gedisa.

Payares-Loyo, L. (2011). Complejidad y humanidades: conciliando el ser y el devenir. Cuadernos del CENDES, 28 (77), 17-42. Recuperado de: http://saber.ucv.ve/ojs/index.php/rev_cc/article/view/3406/3261

Pérez-Luna, E. y Moya-Norys, A. (2008). Diálogo de saberes y proyectos de investigación en la escuela. EDUCERE, 12 (42), 455-460.Recuperado de: http://www.redalyc.org/pdf/356/35614569005.pdf

Pérez-Luna, E., Moya-Norys, A. \& Curcu-Colón, A. (2013). Transdisciplinariedad y educación. Educere, 17 (56), 15-26.Recuperado de: http://www.redalyc.org/pdf/356/35630150014.pdf

Prigogine, I. \& Nicolis, G. (1987). La estructura de lo complejo. Madrid: Alianza Editorial.

Rodríguez-Zoya, L \& Aguirre, J. (2011). Teorías de la complejidad y ciencias sociales. Nuevas Estrategias Epistemológicas y Metodológicas. Nómadas. Revista Crítica de

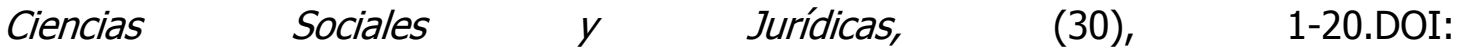
http://dx.doi.org/10.5209/rev_NOMA.2011.v30.n2.36562 
Rodríguez, M. (2017). Currículum, educación y cultura en la formación docente del siglo XXI desde la complejidad. Educación y Humanismo, 19(33), 425-440. DOI: http://dx.doi.org/10.17081/eduhum.19.33.2654

Ross, W. (2012). An Introduction to Cybernetics. Estados Unidos: Filiquarian Legacy Publishing

Shannon, C. (1998) . Teoría matemática de la comunicación. Santiago de Chile: Ediciones Forja.

Thom, R. (1974). Modèlesmathématiques de la morphogénèse. París: U.G.E.

Thom, R. (1988). Esquissed'unesemiophysique. Physiquearistotélicienne et théorie des catastrophes. París: InterEditions.

Von Neumann, J. (1966). Theory of self-reproducing automata. Estados Unidos: University of Illinois Press. Recuperado de: http://fab.cba.mit.edu/classes/862.16/notes/computation/vonNeumann-1966.pdf

Weaver, W. (1948). Science and complexity. American Scientist (36), 536-544. Recuperado de: http://www.filosofiatematica.ufpa.br/index_htm_files/science_and_complexity.pdf

Wiener, N. (1986). Cibernética. Barcelona, España: Edicions Cientifiques Catalanes.

Zapata, O. (2005). Herramientas para elaborar tesis e investigaciones socioeducativas. México: Editorial Pax. 